\title{
Aprovechamiento de un aceite residual siderúrgico en el proceso de sinterización ${ }^{(\bullet)}$
}

\author{
A. Cores ${ }^{(*)}$, A. Formoso $^{(*)}$, A. Moro ${ }^{(*)}$ y P. Marañón ${ }^{(* *)}$ \\ Resumen Se estudia el aprovechamiento de un aceite residual (tinol) previamente utilizado como lubricante en \\ un proceso de laminación. Dicho aceite residual, junto con propano, se emplea como combustible \\ para el encendido de una planta piloto de sinterización. El tinol y el propano se inyectan simultánea- \\ mente por conducciones separadas. Se observa la evolución de la combustión por análisis de $\mathrm{O}_{2}, \mathrm{CO}$ \\ e inquemados en los gases que se producen durante los tres primeros minutos de la sinterización. Se \\ considera efectiva la combustión del tinol y el aporte de energía al proceso, que se manifiesta por una \\ disminución en el consumo de propano a medida que la tasa de tinol inyectado aumenta.
}

Palabras clave: Aceites residuales. Aprovechamiento de residuos. Combustión de residuos. Sinterización.

\section{Exploitation of a siderurgical waste oil in the sintering process}

\begin{abstract}
The exploitation of a waste oil (tinnol) previously used as a lubricant in the rolling process is studied. This waste oil, together with propane, is used as a fuel for the ignition in a sintering pilot plant. Tinnol and propane are simultaneously injected through separate feed lines and the combustion process is monitored by analysis of the levels of $\mathrm{O}_{2}, \mathrm{CO}$ and unburnt particles in the gases produced during the first three minutes of sintering. The combustion of tinnol is considered to be effective and its energy contribution to the process is demostrated by a reduction in the consumption of propane as the rate at which tinnol is injected increases.
\end{abstract}

Keywords: Waste oils. Waste exploitation. Waste combustion. Sintering.

\section{INTRODUCCIÓN}

La preocupación por dar a los residuos peligrosos de origen industrial un destino adecuado, para la preservación de la salud humana y del medio ambiente, es relativamente reciente en los países industrializados. El acusado aumento de la contaminación en los medios receptores y la resonancia mundial de algunos sucesos ampliamente difundidos han favorecido la concienciación ciudadana sobre este problema. A partir del decenio de los 70, los gobiernos y la opinión pública empezaron a prestar atención a las consecuencias medioambientales que supone la inadecuada eliminación o el tratamiento de estos residuos.

La CEE, a través de cuatro programas de acción medioambiental elaborados entre 1973 y 1992, ha

$(\bullet \quad$ Trabajo recibido el día 19 de junio de 1995.

(*) Centro Nacional de Investigaciones Metalúrgicas (CSIC). Avda. de Gregorio del Amo, 8. 28040-Madrid (España).

(**) Corporación Siderúrgica Integral (CSI). Apdo. 90. 33480-Avilés (España). dictado normas para evitar que el medio ambiente se dañe por la eliminación incontrolada de aceites usados, y también para garantizar tanto la eliminación sin riesgos como el reciclado de los residuos.

Las plantas siderúrgicas, y principalmente sus trenes de laminación, producen gran cantidad de aceites residuales que deben eliminarse por incineración o por otros medios puesto que su vertido está prohibido.

En el presente trabajo se estudia una forma de aprovechamiento de estos aceites.

En una planta piloto equipada con un horno de $660 \mathrm{l} / \mathrm{h}$ de capacidad se queman unas emulsiones residuales usadas en laminación en frío, con unos contenidos de aceite del 3 al $10 \%$ y $1.000 \mathrm{~g} / \mathrm{m}^{3}$ de sólidos. Cuando una emulsión, con, un $6 \%$ de aceite, se.inyecta en el horno, sus componentes orgánicos experimentan una combustión muy completa y el contenido de agua se evapora rápidamente. Se consumen entre 130 y $160 \mathrm{~kg}$ de fuel-oil por cada $\mathrm{m}^{3}$ de emülsión tratada (1).

La empresa Cockerill Sambre inyecta en las toberas del horno alto núm. 6 de Lieja, en una tasa de hasta $600 \mathrm{l} / \mathrm{h}$ por tobera, un aceite residual, que 
después de un tratamiento de purificación ha reducido su contenido de agua al $2 \%$ y posee una capacidad calorífica de $42.500 \mathrm{~kJ} / \mathrm{kg}$. En los años 1991 y 1992 se inyectaron en el horno 3.900 y 3.700 t de aceite, respectivamente. Puesto que el aceite estaba casi libre de agua, no se detectaron problemas. En las toberas de los hornos altos núms. 4 y 5 de Charleloi, se inyectaron aceites residuales menos purificados, con un contenido de agua del 35 al $60 \%$, en una tasa de 500 t/año por horno. Este aceite impuro causa problemas en la marcha del horno y, por ello, cada dos días se tiene que cambiar la posición de la tobera donde se hace la inyección, y si la temperatura del arrabio desciende de $1.400{ }^{\circ} \mathrm{C}$, se tiene que parar la inyección de la emulsión (2).

En Alemania se producen anualmente en los trenes de laminación 170.000 t de cascarillas contaminadas de aceite, y en la industria del automóvil $550.000 \mathrm{t}$ de aceite residual. Por el proceso Carbofer las cascarillas aceitosas se mezclan con las cenizas volantes que proceden de una central térmica, y esta mezcla, después de tamizada, es adecuada para el transporte neumático hasta las toberas del horno alto, donde se han inyectado en tasas de $38 \mathrm{~kg}$ de cascarilla aceitosa/t de arrabio. El aceite de automóvil también se ha inyectado en las toberas en tasas de $8 \mathrm{~kg} / \mathrm{t}$ arrabio. En ambos casos, no ha habido alteración de la marcha del horno, y se estima que los volúmenes de inyección se pueden elevar (3).

El objetivo de este trabajo es la utilización y al mismo tiempo la eliminación limpia del tinol (un aceite residual que se utiliza en siderurgia, muy difícil de manipular, almacenar y destruir) mediante su combustión en el horno de encendido de una máquina de sinterización, consiguiendo, además, un beneficio adicional de ahorro de combustible.

La parte experimental del trabajo se ha realizado en la planta piloto de sinterización que el CENIM tiene instalada en Corporación Siderúrgica Integral, CSI, en Avilés.

\section{TINOL}

El tinol es un aceite animal, que se presenta en estado pastoso y tiene una densidad de $0,888 \mathrm{~g} / \mathrm{cm}^{3}$. El termoanálisis del tinol, realizado con un equipo SHIMADZU DTA-50 en las condiciones experimentales de atmósfera de aire, velocidad de flujo 20 $\mathrm{ml} / \mathrm{min}$ y velocidad de calentamiento $10{ }^{\circ} \mathrm{C} / \mathrm{min}$, permite determinar los parámetros que muestra la tabla I.

La composición química del tinol se ofrece en la tabla II. Los sedimentos totales corresponden a los carbonosos más los inorgánicos (cenizas); de los valores de la tabla II se deducen los sedimentos carbonosos u orgánicos, que son 35,76 - 33,37= $2,39 \%$.

TABLA I.- Parámetros del tinol determinados por termoanálisis

TABLE I.- Tinnol parameters fixed by thermoanalysis

Temperatura de fusión, ${ }^{\circ} \mathrm{C}$

Temperatura de inflamación, ${ }^{\circ} \mathrm{C}$

Temperatura de combustión, ${ }^{\circ} \mathrm{C}$

Poder calorífico, $\mathrm{kJ} / \mathrm{kg}$

TABLA II.- Composición química del tinol, \% en masa

TABLE II.- Chemical composition of tinnol, \% in mass

\begin{tabular}{|lr|}
\hline Humedad & 1,02 \\
Materia orgánica & 63,25 \\
Sedimentos totales & 35,76 \\
Cenizas & 33,37 \\
$\mathrm{Cl}^{-}$ & 0,05 \\
$\mathrm{~S}$ & 0,15 \\
$\mathrm{~N}$ & 0,87 \\
\hline
\end{tabular}

En la tabla III se observa el contenido de las materias volatilizables que se producen en la combustión del tinol.

La tabla IV ofrece la composición de las cenizas del tinol, con un contenido de cenizas sulfatadas $<0,2 \%$, indicativo de que su aporte de azufre, por ser muy bajo, no incidirá en la calidad del sinterizado.

\subsection{Aplicación del tinol}

El tinol se utiliza como lubricante de la chapa laminada en el tren tándem. Para su utilización, es necesario emulsionarlo previamente con agua a 85 ${ }^{\circ} \mathrm{C}$, operación que se realiza por agitación con aire en tanques de acero inoxidable. La mezcla de tinol y agua varía en función de los espesores de chapa que se quieren obtener; como referencia, oscila entre 6 y $10 \%$ de tinol. Éste incorpora en origen aditivos químicos que facilitan la posterior separación del agua, una vez que la emulsión entra en contacto con la chapa, para evitar oxidaciones.

TABLA III.- Materias volatilizables en la combustión del tinol, $\mathrm{mg} / \mathrm{kg}$

TABLE III.- Volatilizable elements in the combustion of tinnol, $\mathrm{mg} / \mathrm{kg}$

\begin{tabular}{|cccccccc|}
\hline $\mathrm{Cr}$ & $\mathrm{Ni}$ & $\mathrm{Zn}$ & $\mathrm{Cd}$ & $\mathrm{Pb}$ & $\mathrm{Fe}$ & $\mathrm{Mn}$ & $\mathrm{Cu}$ \\
\hline 2,0 & 47,2 & 24,9 & 2,0 & 18,7 & 355,1 & 83,0 & 6,2 \\
\hline
\end{tabular}


TABLA IV.- Composición química de las cenizas del tinol, \% en masa

TABLE IV.-Chemical composition of tinnol ashes, \% in mass

\begin{tabular}{lrlc|}
\hline $\mathrm{SiO}_{2}$ & 1,90 & $\mathrm{TiO}_{2}$ & 0,24 \\
$\mathrm{Al}_{2} \mathrm{O}_{3}$ & 1,17 & $\mathrm{Na}_{2} \mathrm{O}$ & 0,05 \\
$\mathrm{Fe}_{2} \mathrm{O}_{3}$ & 93,33 & $\mathrm{~K}_{2} \mathrm{O}$ & $<0,01$ \\
$\mathrm{FeO}$ & 0,45 & $\mathrm{ZnO}$ & 0,035 \\
$\mathrm{CaO}$ & 0,70 & $\mathrm{CuO}$ & 0,060 \\
$\mathrm{MnO}$ & 0,12 & $\mathrm{NiO}$ & 0,038 \\
$\mathrm{MgO}$ & 0,01 & $\mathrm{Cr}_{2} \mathrm{O}_{3}$ & $<0,01$ \\
\hline
\end{tabular}

El tinol, una vez utilizado, es un residuo que es necesario eliminar. CSI produce 1.000 t/año de tinol residual y la misma cantidad de otros aceites minerales que se utilizan en circuitos hidráulicos de transmisión, en cárteres de motores de laminación y en aplicaciones similares.

Algunos aceites, como el tinol, son parcialmente regenerados por compañías contratadas con un coste elevado, sólo tolerado por la imposibilidad de deshacerse de una manera fácil y racional de este producto sin inversiones muy costosas, bien en instalaciones de incineración, bien en escombreras impermeabilizadas y especiales. En los últimos años, el descenso del precio de las grasas animales ha hecho disminuir los beneficios de las empresas contratadas para la regeneración del tinol, lo que ocasiona grandes problemas para conseguir compañías que deseen realizar este tipo de trabajos, aceptándolo únicamente cuando consiguen otras compensaciones en otros trabajos complementarios.

En el presente trabajo se pretende eliminar el tinol por combustión en la máquina de sinterización, lo que asegura la combustión completa y la emisión a la atmósfera de los productos finales junto con los que resultan de la combustión del coque y de la descomposición de la caliza del lecho de sínter.

\subsection{Recuperación de tinol}

En la planta de CSI de recuperación de tinol se envía el usado, junto con gran cantidad de agua y lodos, desde el sótano de la nave de laminación hasta el depósito de decantación. La mezcla se envía desde este depósito al tanque decantador de $90 \mathrm{~m}^{3}$, calefactado, donde se decanta por gravedad en tres fases: la superior está formada por tinol, la intermedia es agua y la que se deposita en el fondo está constituida por sedimentos.

El tinol se pasa del tanque de $90 \mathrm{~m}^{3}$ a otro de 60 $\mathrm{m}^{3}$ donde se almacena. En una etapa posterior, el tinol se somete a una purificación final en un circuito de centrifugación.

\section{PROCEDIMIENTO EXPERIMENTAL}

\subsection{Combustión de tinol en la planta piloto de sinterización}

La planta piloto de sinterización en la que se han realizado los ensayos de combustión del tinol, que se muestra en esquema en la figura 1, es de utilización conjunta CENIM-CSI y está instalada en Avilés. Dicha planta dispone de una paila (lecho de sínter) de $420 \times 420 \mathrm{~mm}$ de sección transversal y 400 mm de altura, está equipada con un circuito de succión capaz de crear depresiones superiores a 1.500 $\mathrm{mm} \mathrm{H}_{2} \mathrm{O}$, con un sistema de encendido con gas propano y con un equipo para la medida del volumen de aire de entrada y de temperatura del gas de salida.

Para la realización de los ensayos se modifica la disposición actual de la planta mediante la colocación sobre la paila de una campana de encendido.

\subsection{Construcción de la campana de encendido}

En la máquina industrial de sinterizar, la temperatura de la bóveda en el horno de encendido permanece próxima a $1.200{ }^{\circ} \mathrm{C}$, mientras que en la planta piloto, al ser el proceso discontinuo, se parte de la temperatura ambiente, por lo que será necesario disponer de una nueva campana con recubrimiento refractario calentada a $1.200{ }^{\circ} \mathrm{C}$. De esta manera, al iniciarse la operación el tinol estará a una temperatura semejante a la existente en el encendido industrial.

Con este fin se ha diseñado y construido una campana de encendido (Fig. 2) para adaptar la planta a los ensayos de combustión de tinol. Para el diseño se consultó información facilitada por el IRSID (4) y el CRM (5).

La coraza de la campana está construida con chapa de acero refractario de $4 \mathrm{~mm}$ de espesor, sobre la que se coloca una manta de fibra cerámica de $12,5 \mathrm{~mm}$ de espesor y, a continuación, una capa de ladrillo aislante de $30 \mathrm{~mm}$. Se revistió toda esta superficie con una capa de hormigón de $34 \mathrm{~mm}$ capaz de soportar una temperatura de $1.800{ }^{\circ} \mathrm{C}$.

La campana tiene forma troncopiramidal, cuya base de apoyo mide $420 \mathrm{~mm}$ de lado, para ajustarse a las dimensiones de la paila, y la altura $240 \mathrm{~mm}$. Dispone de cuatro orificios, dos para realizar la inyección del tinol y dos para la medida de la temperatura mediante termopar y pirómetro óptico. La campana está dotada en su parte superior de un orificio que sirve para introducir el quemador, de tipo industrial, de fácil manejo y consumo regulable, 


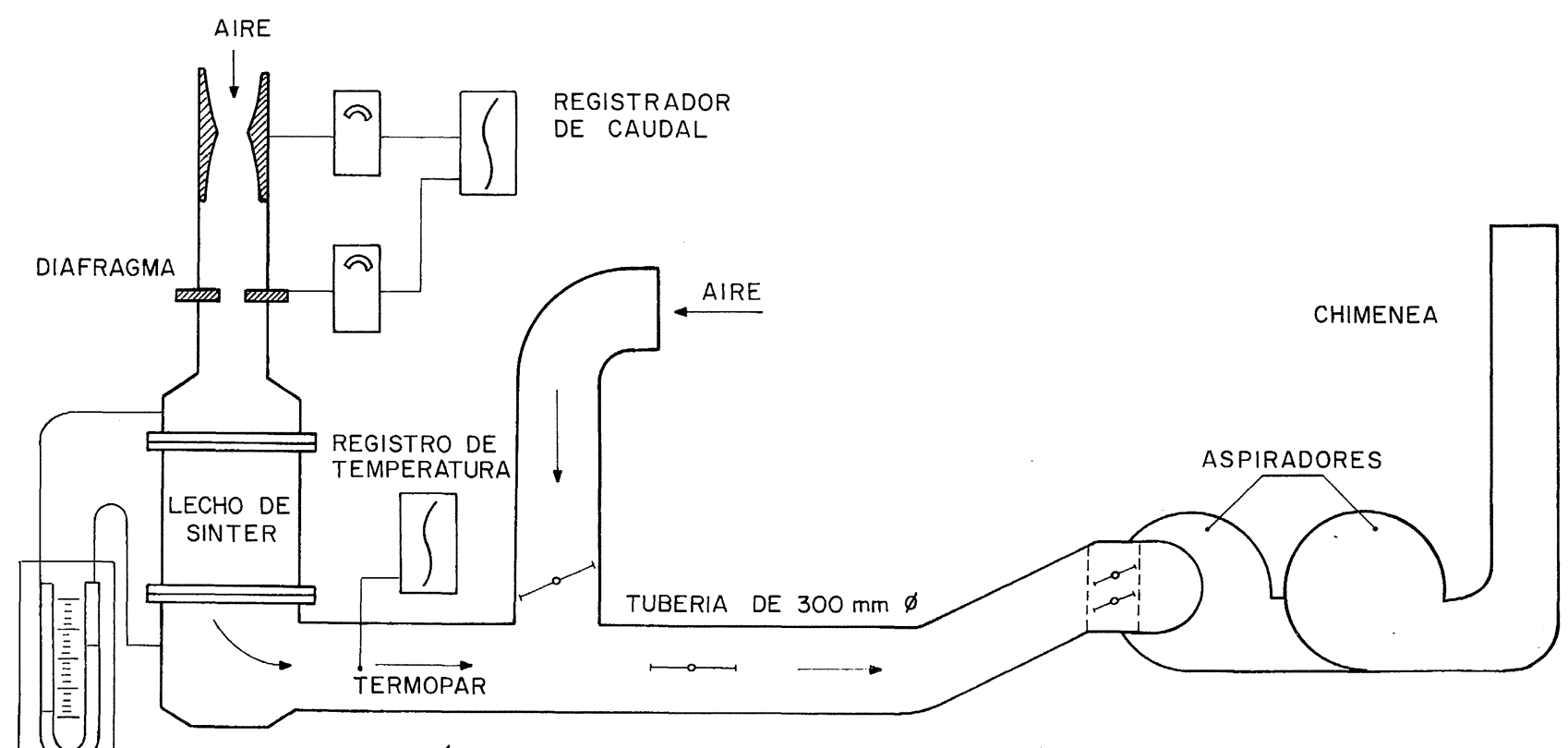

MEDIDA DE LA DEPRESIÓN

EN EL LECHO DE SINTER

FIG. 1.- Esquema de la planta piloto de sinterización.

Fig. 1.- Sintering pilot plant scheme.

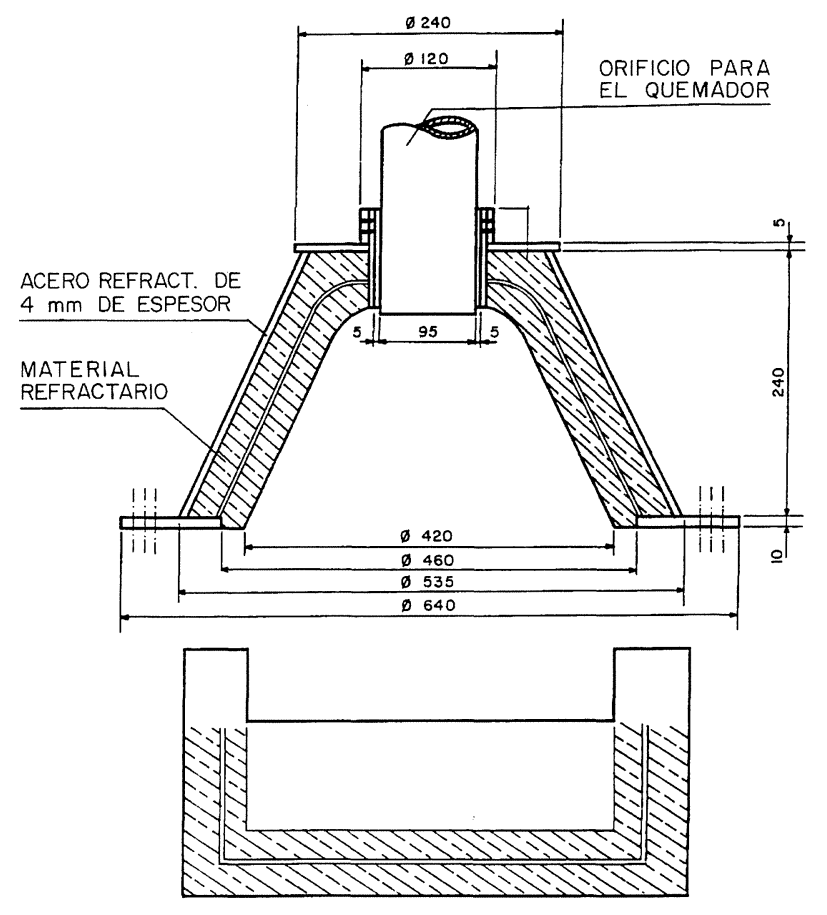

FIG. 2.- Esquema de la campana de encendido.

Fig. 2.-Ignition bell scheme.

con boquilla y mezclador de fundición para hacerlo resistente a las altas temperaturas.

Se ha construido un soporte metálico recubierto de material refractario para apoyo de la campana durante los períodos de calentamiento y de no utilización.

\subsection{Sustitución de propano por tinol}

Se plantean, a continuación, una serie de cálculos, teniendo como base la cantidad de tinol que se necesita eliminar por combustión en una máquina de sinterizar y los poderes caloríficos del tinol y del propano.

\subsubsection{Cantidad de tinol a eliminar}

Se estima en 1.000 t/año el tinol que se necesita eliminar. En el caso de que esta eliminación se produzca por inyección en el encendido de una máquina de sinterizar, sería necesario consumir 1.903 $\mathrm{g} / \mathrm{min}$ de tinol. Para la máquina de sinterizar núm. 5 de CSI, en Avilés, con una zona de ignición de 12 $\mathrm{m}^{2}(4 \times 3 \mathrm{~m})$, se obtiene por cálculo que el tinol a eliminar es $158,6 \mathrm{~g} \cdot \mathrm{m}^{-2} \cdot \mathrm{min}^{-1}$.

En la planta piloto la superficie de encendido es $0,176 \mathrm{~m}^{2}$, por ello, la cantidad de tinol a eliminar deberá ser de $28 \mathrm{~g} / \mathrm{min}$, por lo que en el encendido, que dura 1,5 min, será de $42 \mathrm{~g}$.

\subsubsection{Grado de sustitución}

El poder calorífico del propano es 100.830 $\mathrm{kJ} / \mathrm{m}^{3}$, equivalente a $50.314 \mathrm{~kJ} / \mathrm{kg}$, al ser su densi$\mathrm{dad} 2,004 \mathrm{~kg} / \mathrm{m}^{3}$. Puesto que el poder calorífico del tinol es de $34.140 \mathrm{~kJ} / \mathrm{kg}$, el grado de sustitución del propano por tinol es: 


$$
\frac{34.140 \mathrm{~kJ} / \mathrm{kg}}{50.314 \mathrm{~kJ} / \mathrm{kg}}=0,68
$$

es decir, que $1 \mathrm{~g}$ de tinol sustituye a $0,68 \mathrm{~g}$ de propano, equivalente a 0,339 litros de este gas. Al usar $42 \mathrm{~g}$ de tinol durante $1,5 \mathrm{~min}$ de encendido, se sustituyen 14,24 litros de propano, que equivalen a 570 1/h de éste.

\subsubsection{Tasas de sustitución}

En la sinterización en planta piloto, la carga está a temperatura ambiente, y comienza la sinterización con el aporte de energía al lecho debido a la combustión de propano durante el encendido.

En el presente trabajo, se coloca sobre la carga la campana con una superficie radiante a $1.200^{\circ} \mathrm{C}$, que aporta al lecho una energía calculada en 1.958 $\mathrm{kJ}$ durante los 1,5 min del encendido, equivalentes a $777 \mathrm{l} / \mathrm{h}$ de propano. Como resultado se propone utilizar un consumo máximo de propano de 1.610 1/h y un mínimo de $610 \mathrm{l} / \mathrm{h}$ (por debajo de este caudal no aguanta el encendido debido a la aspiración de $100 \mathrm{~mm} \mathrm{H}_{2} \mathrm{O}$ que es necesario mantener).

La eliminación del tinol residual de CSI equivale a eliminar $42 \mathrm{~g}(47 \mathrm{ml})$ durante los 1,5 $\mathrm{min}$ del encendido en planta piloto, y teniendo en cuenta los datos anteriores se plantea realizar una serie de sustituciones en torno a esta cifra, según se indica en la tabla V.

\subsection{Ensayos de sinterización con inyección de tinol}

Para la inyección de tinol en la campana de encendido se ha construido el inyector de acero cuyo esquema se muestra en la figura 3 . El inyector se calibra para conocer la presión de aire que corresponde a cada caudal de tinol y para comprobar la reproducibilidad de las medidas. El resultado del calibrado se indica en la tabla VI.

El tinol, que se encuentra en un recipiente termostatizado a $75{ }^{\circ} \mathrm{C}$, se trasvasa al inyector que lo introduce en la campana al inicio del ensayo.

TABLA V.- Sustitución de propano por tinol

TABLE V.-Replacement of propane by tinnol

\begin{tabular}{|lrrrrr|}
\hline Propano usado, $\mathrm{l} / \mathrm{h}$ & 1.610 & 1.430 & 1.190 & 920 & 75 \\
Propano sustituido, $1 / \mathrm{h}$ & 0 & 180 & 420 & 690 & 860 \\
Propano sustituido, $\%$ & 0 & 11 & 26 & 43 & 53 \\
Tinol inyectado en & & & & & \\
1,5 min de encendido, $\mathrm{ml}$ & 0 & 15 & 35 & 57 & 71 \\
Tinol inyectado, $1 / \mathrm{h}$ & 0 & 0,60 & 1,38 & 2,28 & 2,82 \\
\hline
\end{tabular}

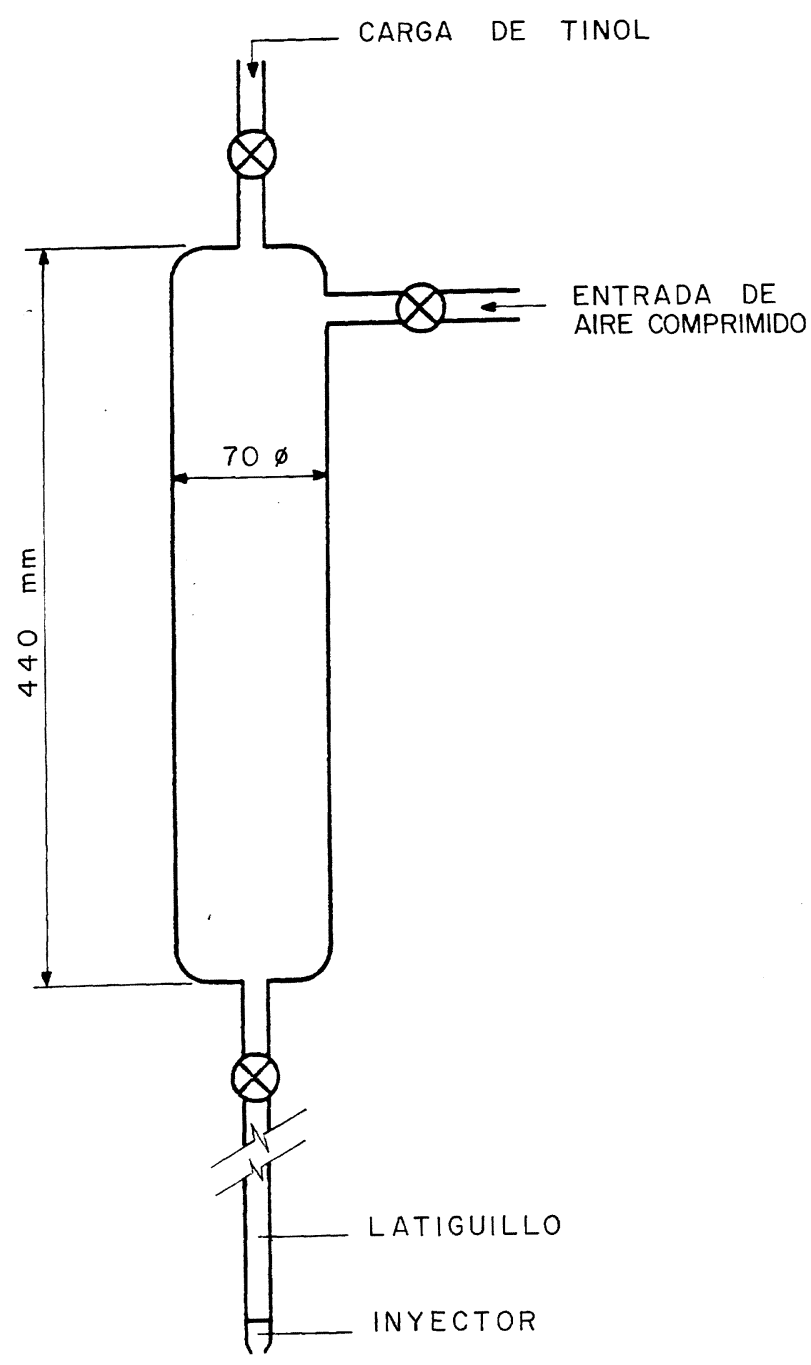

FIG. 3.- Esquema del depósito para la inyección de tinol a presión.

Fig. 3.-Tank for pressure injection of tinnol scheme.

Otras condiciones para realizar los ensayos son las siguientes:

- Tiempo de encendido por combustión de propano: $3 \mathrm{~min}$ (1,5 $\mathrm{min}$ de encendido más $1,5 \mathrm{~min}$ adicionales).

TABLA VI.- Condiciones de trabajo del inyector

TABLE VI.- Working conditions of the injector

\begin{tabular}{|l|cc|}
\cline { 2 - 3 } \multicolumn{1}{c|}{} & $\begin{array}{l}\text { Presión del aire, } \\
\mathrm{kg} / \mathrm{cm}^{2}\end{array}$ & $\begin{array}{l}\text { Tinol inyectado } \\
\text { en 1,5 min, } \mathrm{ml}\end{array}$ \\
\hline Boquilla 1 & 1 & 15 \\
& 4,2 & 35 \\
Boquilla 2 & 2,4 & 57 \\
& 2,8 & 71 \\
\hline
\end{tabular}

Rev. Metal. Madrid, 32 (I), 1996 29

http://revistademetalurgia.revistas.csic.es 
- Tiempo de inyección de tinol: 3 min (1,5 min de encendido más 1,5 min adicionales).

- Depresión en el encendido: $100 \mathrm{~mm} \mathrm{H}_{2} \mathrm{O}$.

- Depresión de trabajo: $1.500 \mathrm{~mm} \mathrm{H}_{2} \mathrm{O}$.

- Altura total del lecho: $400 \mathrm{~mm}$.

- Altura de sobreparrilla: $30 \mathrm{~mm}$.

- Temperatura de la campana al inicio del ensayo: $1.200^{\circ} \mathrm{C}$.

- Mezcla que se carga a la paila para cada ensayo, $\mathrm{kg}$ :

mezcla mineral 100

finos de retorno 25

cal 2

coque 4

La mezcla se homogeneiza con la adición de un $7 \%$ de agua.

- La determinación de $\mathrm{O}_{2}, \mathrm{CO}$ e inquemados se realiza en continuo durante los tres primeros minutos de cada ensayo, en la conducción situada a la salida de la paila.

$\mathrm{El} \mathrm{O}_{2}$ y el $\mathrm{CO}$ se determinan con un analizador digital AUROTRONICS, con medida directa de la concentración de $\mathrm{O}_{2}$ de 0 a 20,9\% y de $\mathrm{CO}$ de 0 a $19.999 \mathrm{ppm}$, a una temperatura de gases de -20 a $1.000^{\circ} \mathrm{C}$.

La determinación de inquemados se realiza con un analizador LEEDS NORTHRUP. Se consideran inquemados las sustancias como el hidrógeno, monóxido de carbono e hidrocarburos, susceptibles de reaccionar con el oxígeno. Para el calibrado del equipo se utiliza un gas patrón con un contenido de inquemados conocido: $1 \% \mathrm{H}_{2}$ y $4 \% \mathrm{CO}$.

\section{RESULTADOS}

Se han realizado 20 ensayos de sinterización, sin y con adición de tinol. La temperatura de la campana en el momento de la inyección de propano (o de propano y tinol), ha sido de $1.200{ }^{\circ} \mathrm{C}$. En la tabla VII se ofrecen los valores medios de $\mathrm{O}_{2}, \mathrm{CO}$ e inquemados analizados en los gases a la salida de la paila, a intervalos de $20 \mathrm{~s}$.

\subsection{Evolución del oxígeno}

La figura 4, construida a partir de valores de la tabla VII, muestra cómo evoluciona el contenido de oxígeno durante los tres primeros minutos del proceso (etapa de encendido) en los gases de combustión. Se observa una disminución en el valor porcentual de $\mathrm{O}_{2}$ con el tiempo, siendo este porcentaje superior para tasas de tinol inyectado mayores. Al final de los $3 \mathrm{~min}$, se obtienen unos niveles de oxígeno bajos (entre 3,5 y $6 \%$ ) como consecuencia del consumido en las combustiones de coque, propano y tinol.

\subsection{Evolución del monóxido de carbono}

La figura 5 muestra cómo evoluciona el contenido de $\mathrm{CO}$ en los gases; se puede apreciar que hay poca variación para las distintas tasas de inyección y que existe un aumento lineal del valor porcentual de CO con el tiempo.

TABLA VII.- Ensayos de sinterización

TABLE VII.- Sintering tests

\begin{tabular}{|c|c|c|c|c|c|c|c|c|c|c|c|c|}
\hline \multirow{2}{*}{$\begin{array}{l}\text { Ensayo } \\
\text { núm. }\end{array}$} & \multirow{2}{*}{$\begin{array}{l}\text { Tinol } \\
\text { injectado, } \\
1 / \mathrm{h}\end{array}$} & \multirow{2}{*}{$\begin{array}{l}\text { Propano } \\
\text { usado, } \\
\text { 1/h }\end{array}$} & \multicolumn{10}{|c|}{ Evolución de la composición en \% de los gases analizados después de un período de } \\
\hline & & & & $20 \mathrm{~s}$ & $40 \mathrm{~s}$ & $60 \mathrm{~s}$ & $80 \mathrm{~s}$ & $100 \mathrm{~s}$ & $120 \mathrm{~s}$ & $140 \mathrm{~s}$ & $160 \mathrm{~s}$ & $180 \mathrm{~s}$ \\
\hline $1-6$ & 0 & 1.610 & $\begin{array}{l}\mathrm{O}_{2} \\
\mathrm{CO} \\
\text { inquemados }\end{array}$ & $\begin{array}{l}14,0 \\
\text { ND } \\
0,25\end{array}$ & $\begin{array}{l}9,5 \\
0,21 \\
0,49\end{array}$ & $\begin{array}{l}6,3 \\
0,36 \\
0,56\end{array}$ & $\begin{array}{l}4,8 \\
0,67 \\
0,75\end{array}$ & $\begin{array}{l}3,9 \\
0,94 \\
0,94\end{array}$ & $\begin{array}{l}3,6 \\
1,21 \\
1,22 \\
\end{array}$ & $\begin{array}{l}3,4 \\
1,34 \\
1,45\end{array}$ & $\begin{array}{l}3,5 \\
1,40 \\
1,68\end{array}$ & $\begin{array}{l}3,2 \\
1,56 \\
1,81\end{array}$ \\
\hline $7-9$ & 0,60 & 1.430 & $\begin{array}{l}\mathrm{O}_{2} \\
\mathrm{CO} \\
\text { inquemados }\end{array}$ & $\begin{array}{l}8,4 \\
0,20 \\
0,15\end{array}$ & $\begin{array}{l}5,9 \\
0,25 \\
0,34\end{array}$ & $\begin{array}{l}4,6 \\
0,48 \\
0,60\end{array}$ & $\begin{array}{l}4,1 \\
0,72 \\
0,93\end{array}$ & $\begin{array}{l}3,7 \\
0,91 \\
1,15\end{array}$ & $\begin{array}{l}3,4 \\
1,03 \\
1,41\end{array}$ & $\begin{array}{l}3,3 \\
1,16 \\
1,58\end{array}$ & $\begin{array}{l}3,3 \\
1,27 \\
1,73\end{array}$ & $\begin{array}{l}3,3 \\
1,41 \\
1,82\end{array}$ \\
\hline $10-12$ & 1,38 & 1.190 & $\begin{array}{l}\mathrm{O}_{2} \\
\mathrm{CO} \\
\text { inquemados }\end{array}$ & $\begin{array}{l}9,0 \\
0,27 \\
0,20\end{array}$ & $\begin{array}{l}6,3 \\
0,36 \\
0,40\end{array}$ & $\begin{array}{l}5,0 \\
0,62 \\
0,56\end{array}$ & $\begin{array}{l}4,4 \\
0,79 \\
0,86\end{array}$ & $\begin{array}{l}4,1 \\
0,98 \\
1,11\end{array}$ & $\begin{array}{l}3,7 \\
1,07 \\
1,27\end{array}$ & $\begin{array}{l}3,6 \\
1,29 \\
1,40\end{array}$ & $\begin{array}{l}3,6 \\
1,47 \\
1,62\end{array}$ & $\begin{array}{l}3,5 \\
1,65 \\
1,65\end{array}$ \\
\hline $13-15$ & 2,28 & 920 & $\begin{array}{l}\mathrm{O}_{2} \\
\mathrm{CO} \\
\text { inquemados }\end{array}$ & $\begin{array}{l}\text { ND } \\
\text { ND } \\
0,35\end{array}$ & $\begin{array}{l}9,2 \\
0,32 \\
0,61\end{array}$ & $\begin{array}{l}7,7 \\
0,57 \\
0,71\end{array}$ & $\begin{array}{l}6,6 \\
0,77 \\
1,22\end{array}$ & $\begin{array}{l}5,9 \\
0,88 \\
1,40\end{array}$ & $\begin{array}{l}5,3 \\
1,00 \\
1,60\end{array}$ & $\begin{array}{l}5,1 \\
1,19 \\
1,69\end{array}$ & $\begin{array}{l}4,9 \\
1,30 \\
1,81\end{array}$ & $\begin{array}{l}4,7 \\
1,49 \\
2,11\end{array}$ \\
\hline $16-20$ & 2,82 & 750 & $\begin{array}{l}\mathrm{O}_{2} \\
\mathrm{CO} \\
\text { inquemados }\end{array}$ & $\begin{array}{l}11,9 \\
\mathrm{ND} \\
0,50\end{array}$ & $\begin{array}{l}9,7 \\
0,29 \\
0,68\end{array}$ & $\begin{array}{l}8,5 \\
0,51 \\
1,01\end{array}$ & $\begin{array}{l}7,3 \\
0,68 \\
1,06\end{array}$ & $\begin{array}{l}6,9 \\
0,85 \\
1,21\end{array}$ & $\begin{array}{l}6,2 \\
1,04 \\
1,51\end{array}$ & $\begin{array}{l}5,9 \\
1,22 \\
1,60\end{array}$ & $\begin{array}{l}5,9 \\
1,37 \\
2,01\end{array}$ & $\begin{array}{l}5,7 \\
1,52 \\
2,02\end{array}$ \\
\hline
\end{tabular}

$\mathrm{ND}=$ No determinado 


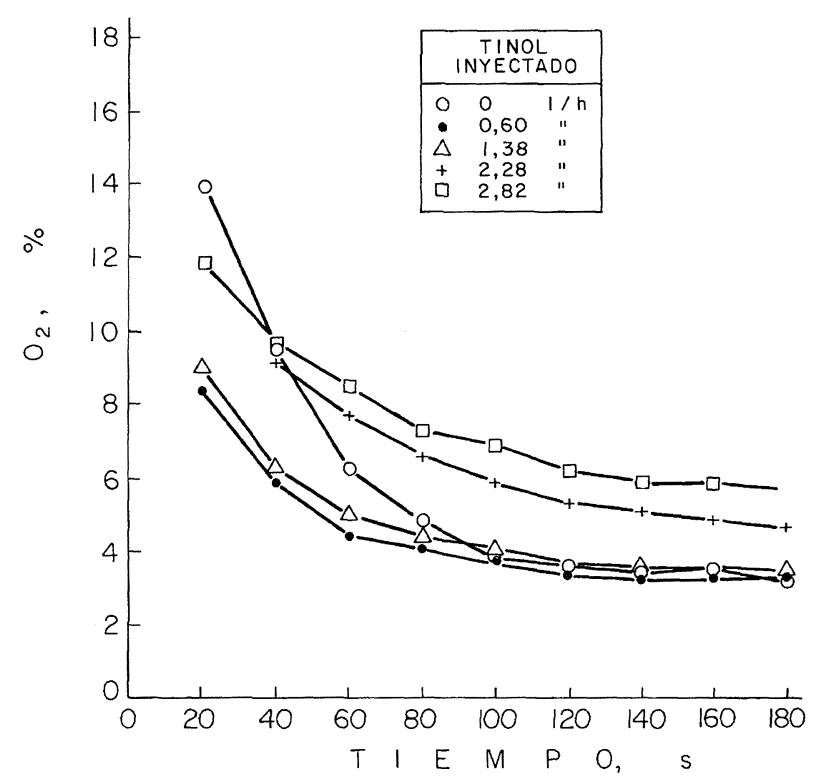

FIG. 4.- Evolución del contenido de oxígeno en los gases de combustión.

Fig. 4.- Evolution of oxygen content of the combustion gases.

\subsection{Evolución de los inquemados}

La figura 6 muestra cómo varía el contenido de inquemados con el tiempo. De forma análoga al $\mathrm{CO}$, existe un aumento lineal de los inquemados con el tiempo, y para tasas mayores de inyección de tinol se aprecia un contenido algo superior de inquemados.

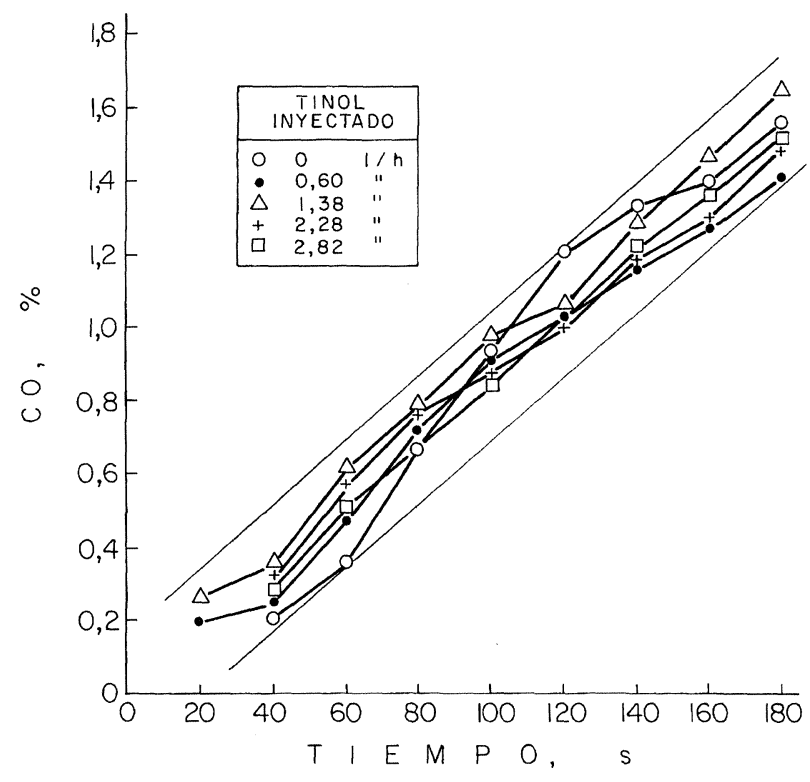

Fig. 5.- Evolución del contenido de monóxido de carbono en los gases de combustión.

Fig. 5.- Evolution of carbon monoxide content of the combustion gases.

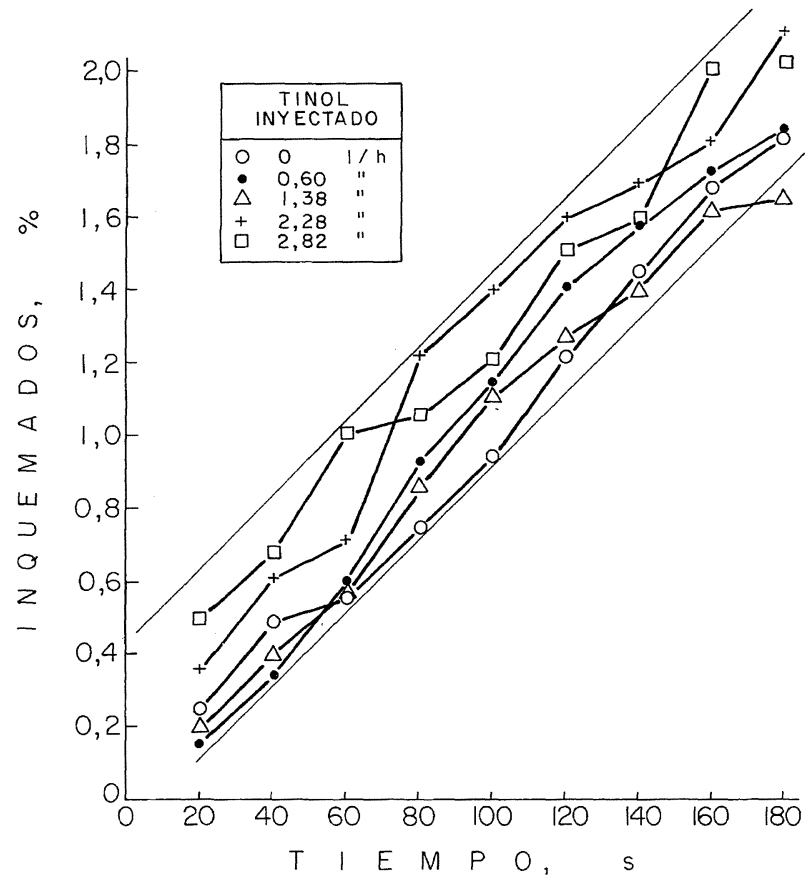

FIG. 6.- Evolución del contenido de inquemados en los gases de combustión.

Fig. 6.- Evolution of unburnt particles content of the combustion gases.

\subsection{Sinterizados fabricados}

Se han realizado los seis primeros ensayos sin inyección de tinol y los catorce restantes con inyección de este aceite. Después de cada ensayo, se realiza el análisis químicos de cada sinterizado, obteniéndose una composición bastante uniforme para todos los sinterizados.

\subsection{Ahorro de consumo de propano}

En las experiencias realizadas se procede a disminuir el consumo de propano a medida que se inyecta tinol. Para una tasa de inyección de 2,82 1/h de tinol, se consigue un ahorro del $53 \%$ de propano con relación al que se consume sin inyección de tinol, como se puede apreciar en la tabla VII.

\section{CONCLUSIONES}

En una planta piloto de sinterización se elimina por combustión el tinol, un aceite animal utilizado como lubricante en laminación. Se ha construido una campana para adaptarla a la instalación de sinterización, y acondicionada para la inyección de propano y tinol. Se realiza la sinterización de la mezcla mineral y se analiza el contenido de $\mathrm{O}_{2}, \mathrm{CO}$ 
e inquemados en los gases de combustión. Los resultados permiten establecer las siguientes conclusiones:

- El tinol experimenta su combustión, con aporte de energía al proceso, que se manifiesta por una disminución del propano utilizado a medida que aumenta la tasa de tinol inyectado. No se produce alteración en la composición del sínter producido.

- El nivel de inquemados que se emite a la atmósfera a través de los humos que se producen durante el encendido en la sinterización no varía, tanto si se usan porcentajes de tinol variables como si se utiliza el propano como combustible convencional.

- Las características propias de la sinterización hacen que los gases de combustión procedentes del encendido atraviesen un lecho de mezcla mineral de 40-60 $\mathrm{cm}$ de espesor, que actúa como filtro primario, a la vez que refrigera los gases con una eficacia muy alta. Otros métodos de incineración de aceites residuales, por el contrario, eliminan los gases de manera más directa con mayor calor sensible.

- Teniendo en cuenta las consideraciones anteriores, y de acuerdo con la normativa legal que regula la incineración de aceites y los niveles permitidos de emisiones a la atmósfera, se puede considerar correcta la eliminación de tinol por combustión en el encendido de la máquina de sinterización.

\section{Agradecimiento}

Los autores agradecen a CSI, Ministerio de Industria y CEDETI el apoyo económico a este Proyecto núm. 1368/92.

\section{REFERENCIAS}

(1) Ebert, R., Braun, H., Klinkhart, A. y Odenstein, C. Neue Hütte, 20 (12), 1975: 742-746.

(2) COCKERILL SAMBRE. Recycling of oily wastes at Cockerill Sambre. Comunicación privada, 1993.

(3) Buchwalder, J., Hunger, J., Kalinowski, W., Krüger, W. y LETZEL, D. Utilization of oil-contaminated millscale and shredder light fraction from automobiles in blast furnaces. Seminar on the Steel Industry and Recycling. Düsseldorf (R.F.A.), 24-27 Abr. 1995.

(4) Meunier, G. Stahl Eisen, 85 (15), 1965: 897-901.

(5) Rochas, R. y Boucraut, M. Rev. Ind. Miner., 48 (4), 1966: 277-292. 